\title{
Contrast discrimination under temporally varying contrast conditions
}

\author{
JAMES L. DANNEMILLER \\ University of Wisconsin, Madison, Wisconsin \\ and \\ BENJAMIN R. STEPHENS \\ Clemson University, Clemson, South Carolina
}

\begin{abstract}
Psychophysical contrast discrimination of a 0.8 -cpd vertical grating was tested using a paradigm that alternated test and masking gratings at $8 \mathrm{~Hz}$. Masking contrasts were lower than, equal to, or higher than the test contrasts. Six test contrasts were combined factorially with six masking contrasts to generate a series of six contrast increment threshold versus test contrast curves (tvc curves). A particularly simple relationship existed between these curves. The curves could be brought into alignment by shifting them diagonally by the ratio of their masking contrasts. It is shown that this behavior is predicted by a model in which contrast gain is set by the average of the test and masking contrasts coupled with a simple model of contrast discrimination. Contrast gain control integrates contrast over a period of at least $125 \mathrm{msec}$, and contrast discrimination is a function of this time-averaged contrast.
\end{abstract}

One of the primary goals of spatial vision is to represent environmental features such as size, orientation, location, and phase in an unambiguous fashion. For some time, we have known that visual cortical neurons respond selectively to these features, and that this stimulus-specific response might provide a method for representing pertinent features in the visual system (Hubel \& Wiesel, 1968). However, the response of a cell could be ambiguous for two reasons. First, a moderate response rate could signal a spatially optimal stimulus with moderate contrast or a spatially nonoptimal stimulus of high contrast. Second, consider the stimulus information as represented in the ratio of two or more cells' responses. The saturation of a cell's response with increased contrast, in conjunction with a limited dynamic range, would change the ratio of the cells' responses and thus render their relative activity ambiguous with regard to the stimulus features. These ambiguities are present if one assumes that a cell's contrast response function is a fixed function of stimulus contrast. However, this assumption may not be valid. Recent physiological evidence indicates that simple cells in the visual cortex adjust their contrast response functions to the contrast of a scene, averaged over space and time (Albrecht \& Geisler, 1991; Bonds, 1989, 1993; Geisler \& Albrecht, 1992; Heeger, 1992a, 1992b, 1994). Both Albrecht and Geisler (1991) and Heeger (1994) have argued that this contrast

This research was supported by a University of Wisconsin Vilas Foundation Fellowship to J.L.D. Portions of this work were presented at the annual meeting of the Association for Research in Vision and Ophthalmology, Ft. Lauderdale, May 1997. Correspondence should be addressed to J. L. Dannemiller, Room 515, Waisman Center, University of Wisconsin, 1500 Highland Ave., Madison, WI 53705-2280 (e-mail: jldannem@facstaff.wisc.edu). gain control offers the advantage of normalizing the cells' responses to stimulus contrast and hence preserves the stimulus-specific response property of cortical neurons. Indeed, recent models of pattern identification indicate that the normalization of cells' responses may allow fairly unambiguous representation of size, orientation, phase, and location in populations of neurons in primary visual cortex (Geisler \& Albrecht, 1997).

Contrast discrimination tasks have been used to examine the operation of contrast-set gain control behaviorally. In a typical contrast discrimination task the observer is required to discriminate two patterns that differ only in contrast (i.e., the test contrast, $c$, and the test plus an increment, $c+\Delta c$ ). Bowen and Wilson (1994) showed that contrast increment threshold for a brief $(30-\mathrm{msec})$ increment was elevated by a background contrast differentially depending on the stimulus onset asynchrony (SOA) between the increment and the background contrasts. For 0 msec SOA, threshold elevation was roughly $1 \log$ unit, and threshold elevation declined quickly over 60 to $200 \mathrm{msec}$ of SOA. This result could be explained by a contrast-set gain control that adapts to stimulus contrast over the course a few hundred milliseconds. In a previous study, we used a somewhat different stimulus, depicted in Figure 1, to examine the effects of contrast-set gain control in a contrast discrimination task (Dannemiller \& Stephens, 1998). Two 0.8-cpd targets ( $c$ or $c+\Delta c$ ) were presented in 1-sec intervals in a two-interval forced-choice task. Each target alternated at $8 \mathrm{~Hz}$ with various masking stimuli (i.e., a uniform field, a masking grating with contrast $c$, a masking grating with contrast $c$ with random spatial phase shifts on each cycle, or a masking grating with contrast greater than the test grating contrast, $c$ ). One advantage of the 8$\mathrm{Hz}$ alternation of mask and target is that both forward and 


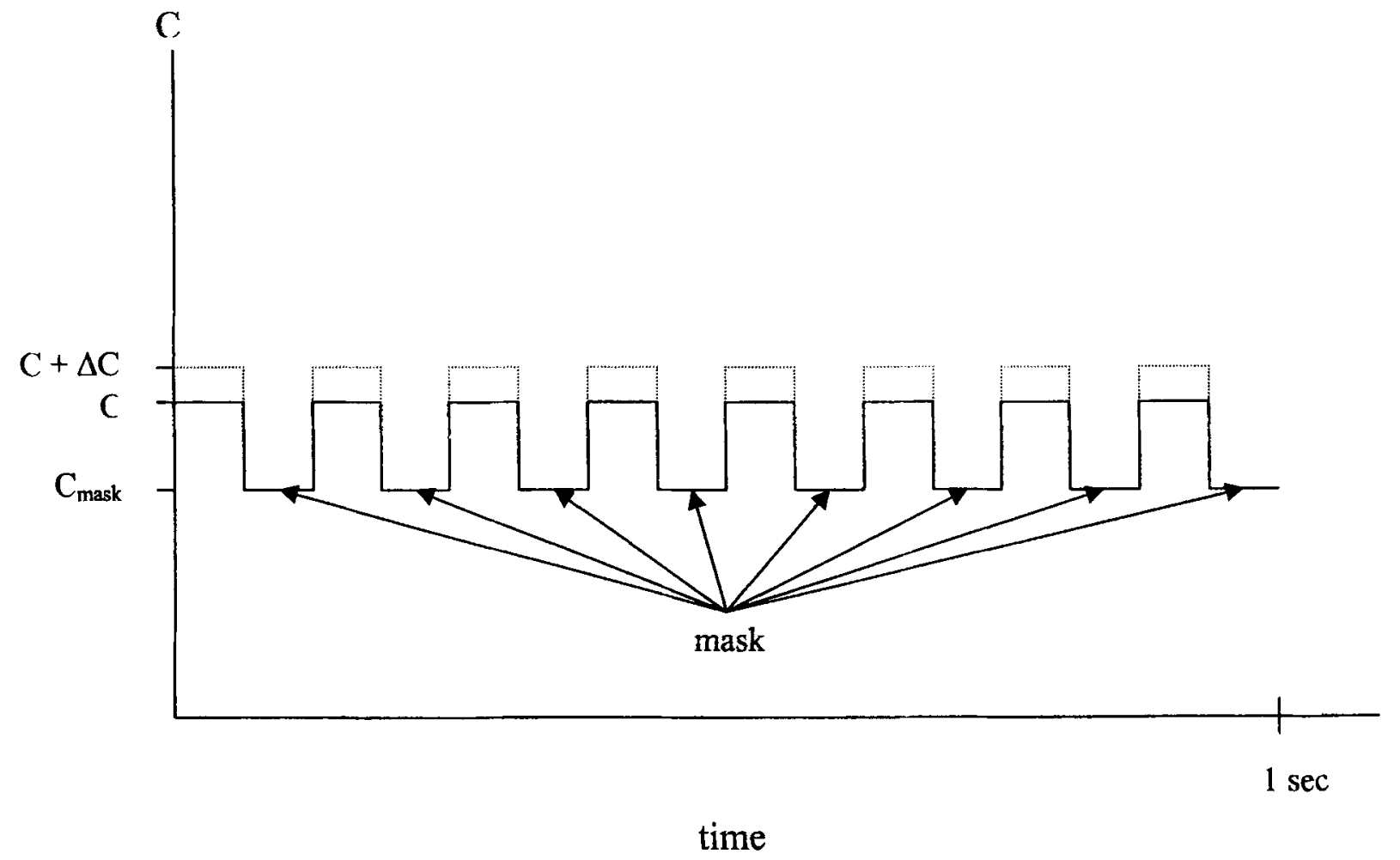

Figure 1. Temporal characteristics of the stimulation. The test grating at a contrast of $c$ or $c+\Delta c$ alternated at $8 \mathrm{~Hz}$ (square wave) with the masking grating at a contrast of $c_{\text {mask }}$. A two-temporal alternative forced-choice procedure was used with the stimulation lasting 1 sec in each of the two intervals. The intervals were separated by 1 sec. The observer's task was to say which interval contained the test grating with the larger contrast. In this schematic, the mask contrast is less than the test contrast. In other conditions, the mask contrast was zero, equal to, or greater than the test contrast.

backward masking stimuli are represented in the task. Georgeson and Georgeson (1987) have demonstrated that forward and backward masking have similar influences on performance in a contrast discrimination task, and we assume that our stimuli generate both masking effects. It is plausible to argue that ours is an ecologically valid stimulus because normal visual tasks are often embedded in a stream of contrast-varying stimulation.

In general, the results of our previous study indicate that alternating the targets with stimuli other than the identical background grating $(c)$ increases increment threshold. This result may reflect the operation of contrast-set gain control. At the $8-\mathrm{Hz}$ alternation rate, the target is available for only $62.5 \mathrm{msec}$ before shifting to a different stimulus contrast (e.g., a uniform field or a higher contrast grating). Thus gain control is not able to adapt the contrast response function to the target contrast. One consequence of this incomplete adaptation to target contrast is that the steepest portion of the response function is not positioned at $c$, and thus $\Delta c$ is elevated.

One implication of our previous data is that very rapid shifts in stimulus contrast can cause gain control to adjust the response functions of populations of neurons to an effective contrast that is equal approximately to the recent time-averaged contrasts. The goal of the present experi- ment was to evaluate this simple hypothesis systematically by alternating the targets $(c$ or $c+\Delta c)$ at $8 \mathrm{~Hz}$ with masking stimuli of various contrasts and to estimate the effects of this alternation on contrast discrimination functions. The task that we used was designed to examine contrast discrimination under conditions like those that obtain in everyday vision. Eye movements that occur at an approximate rate of three to four per second produce temporally varying contrasts over local retinal regions. It is of interest to determine how contrast discrimination is affected by such temporal contrast variation.

In the context of a model of contrast discrimination that includes a simple gain control feature, when the test and masking contrasts differ our stimuli should induce gain control to position the contrast response function somewhere between the test contrast present in the first half of each $8-\mathrm{Hz}$ cycle and the masking contrast present in the last half of each cycle. Thus, when the two contrasts are equal, the response function should be optimally positioned and $\Delta c$ should be near its minimum. When the masking and test contrasts are not equal, $\Delta c$ should be elevated. We test a model below in which the masking and test contrasts are linearly averaged to set the contrast gain, which in turn determines the sensitivity to incremental contrast. 


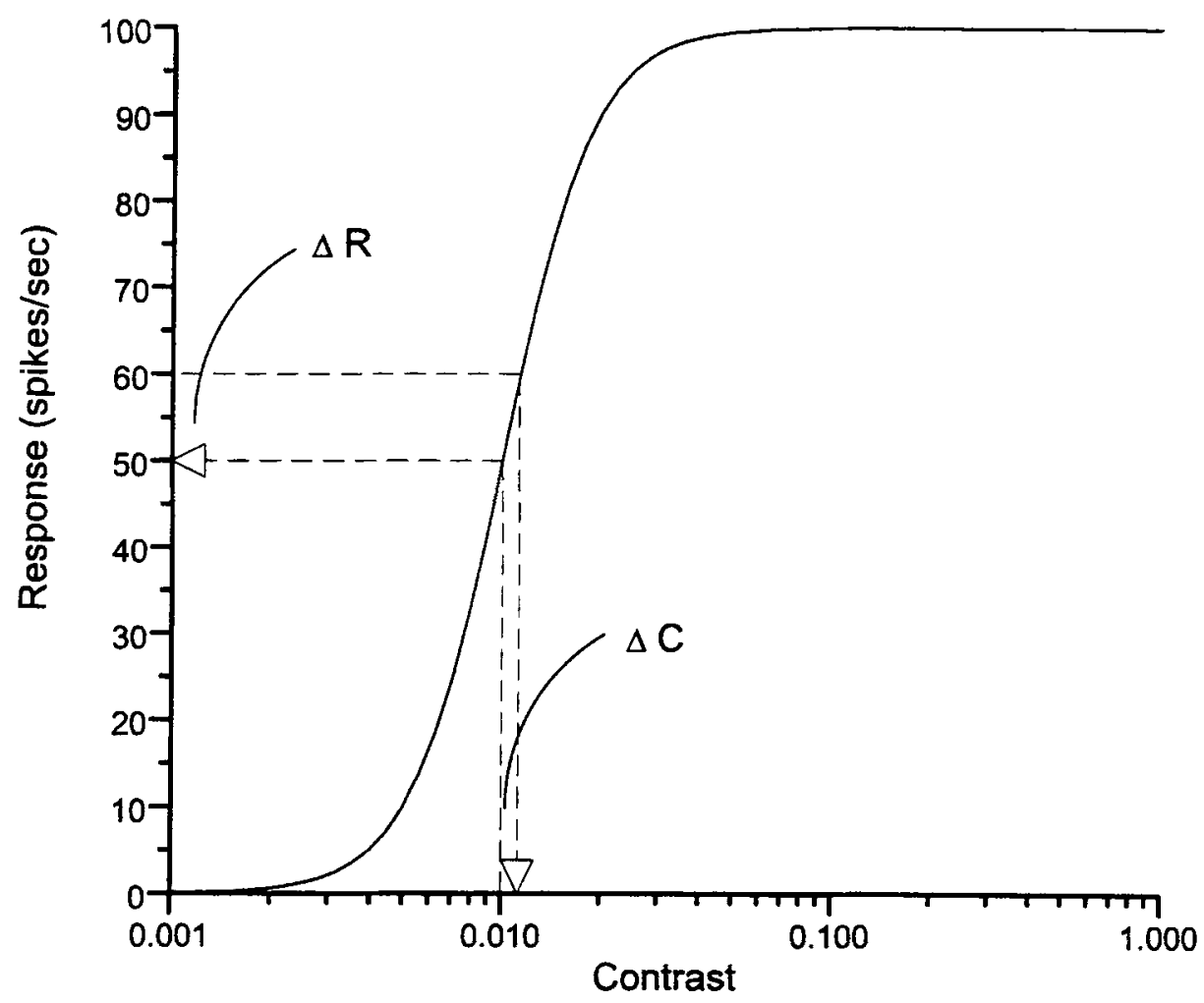

Figure 2. Plot of response versus contrast using Equation 1. Threshold was defined as the increment in contrast, $\Delta c$, necessary to evoke a constant criterion change in responding, $\Delta R$.

\section{METHOD}

\section{Subjects}

Four observers provided estimates of increment thresholds for each of the conditions described below. Two observers provided three estimates per condition, and the other 2 provided one estimate per condition. All were trained and had normal or corrected-to-normal acuity.

\section{Apparatus and Stimuli}

Vertical sine-wave gratings were computer generated using a Vision Metrics PC Pattern Generator, resident in an IBM PC, and displayed on an INFAX (DM/9B) composite monitor. The PC Pattern Generator controls signal contrast through a linear attenuator ( 128 levels) and an independent logarithmic attenuator (256 levels) that are cascaded to allow precise contrast control. Cascading these attenuators provided 32,768 possible luminance levels. The monitor was calibrated using a UDT (151) photometer. The maximum calibrated contrast on the monitor was 0.72 . The mean luminance was $68 \mathrm{~cd} / \mathrm{m}^{2}$. Observers viewed the display from a distance of $84 \mathrm{~cm}$. The 0.8 -cpd gratings were presented through a $10^{\circ} \times 8^{\circ}$ aperture, and the surround was dark.

Displays were presented at central fixation as follows. The test grating ( $c$ or $c+\Delta c$ ) alternated at a temporal rate of $8 \mathrm{~Hz}$ (square wave) with the masking grating. See Figure 1 for the temporal conditions of stimulus presentation. It is the test periods of each temporal cycle that contain the test gratings relevant to the contrast discrimination. The mask periods contain the masking gratings, which were always constant across both test intervals. If contrast discrimination were determined at least in part by a contrast-set gain control mechanism that integrated contrast across both the test and the mask periods, then discrimination should be strongly affected by the masking contrast in the mask intervals, especially when the test and masking contrasts differ substantially in contrast. An additional goal of the present experiment was to characterize quantitatively how this masking contrast affects contrast discrimination.

\section{Design and Procedure}

Six different test contrasts were combined with six different masking contrasts to create 36 conditions. The test and masking contrasts were $.00, .006, .022, .067, .2$, and .6 . Observers were tested on these 36 conditions in different random orders.

The observer's task in all conditions was to choose the interval that contained the test grating with the greater contrast. A two-temporal interval forced-choice procedure was used to display the gratings. One interval contained the test grating at contrast $c$ alternating at $8 \mathrm{~Hz}$ with the masking grating, and the other interval contained the test grating at contrast $c+\Delta c$, also alternating at $8 \mathrm{~Hz}$ with the same masking grating. Each interval was $1 \mathrm{sec}$ long and the intervals were separated by $1 \mathrm{sec}$. The spatial phase of the grating with respect to the center of the display was randomized before each trial to reduce potential effects associated with long-term local luminance adaptation. The contrast difference between the gratings was controlled by a 3-down, 1-up staircase, with a step size of $2.0 \mathrm{~dB}$. The initial value of $\Delta c$ was set by the observer to a contrast level noticeably above threshold. Six reversals were used to estimate increment threshold by averaging the logs of the reversal contrasts.

\section{Modeling}

The contrast increment threshold data were modeled using a contrast response function (crf) that has been used previously to model the responses of cortical neurons to contrast (Albrecht \& Hamilton, 


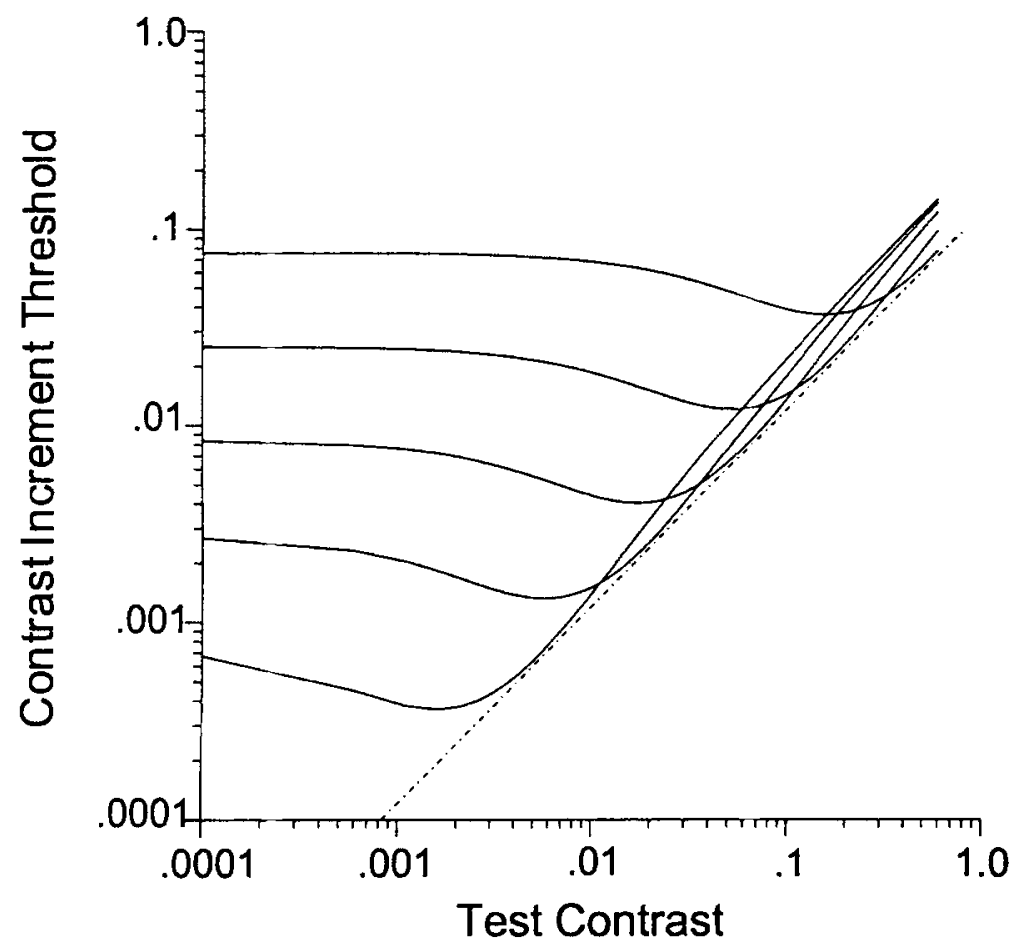

Figure 3. Equation 4 plotted for various combinations of fest ( $x$-axis) and masking contrasts. Each curve represents a different fixed masking contrast alternated with a series of test contrasts on the $x$-axis. An exponent of 2.0 was used for the nonlinearity, and a criterion response change of 6 spikes per second ( $6 \%$ of maximum response) was used to solve for the threshold contrast increment, $\Delta c$, plotted on the $y$-axis. All of these curves shift up and to the right along a diagonal line (dashed) by the ratio of their masking contrasts as the masking contrast is increased. They dip in the region where the masking and test contrasts equal each other.

1982; Heeger, 1992a, 1992b; Sclar, Maunsell, \& Lennie, 1990). This crf is shown in Equation 1.

$$
R(c)=R_{\max } \cdot\left[\frac{c^{n}}{c^{n}+\sigma^{n}}\right] .
$$

In Equation 1, $R$ stands for the response of the neuron in spikes per second, $R_{\max }$ is the maximum firing rate (arbitrary; assumed to be 100), $c$ is test contrast, $n$ is the exponent on the contrast versus response nonlinearity, and $\sigma$ is the contrast at which the neuron shows half maximum response. Changes in $\sigma$ shift the crf laterally along a logarithmic contrast axis, leaving its shape invariant (Heeger, 1992b). Equation 1 is the Naka-Rushton equation that was used first to model the responses of photoreceptors to different intensities of light (Naka \& Rushton, 1966).

Threshold was modeled as the increase in contrast, $\Delta c$, necessary to evoke a fixed criterion increase in the response, $\Delta R$. This increment in contrast is shown in Equation 2.

$$
R(c+\Delta c)=R_{\max } \cdot\left[\frac{(c+\Delta c)^{n}}{(c+\Delta c)^{n}+\sigma^{n}}\right]
$$

This increment in contrast leads to an increment in the response, as shown in Figure 2 and Equation 3:

$$
\Delta R=R(c+\Delta c)-R(c) .
$$

Equations 2 and 3 can be solved for the $\Delta c$ necessary to evoke a criterion increase in response, $\Delta R$. When this is done, Equation 4 results:

$$
\Delta c=\left[\frac{\sigma^{n}}{\frac{1}{\frac{\Delta R}{R_{\max }}+\frac{c^{n}}{c^{n}+\sigma^{n}}}-1}\right]^{\frac{1}{n}}-c
$$

The model that we are using here was first used to model threshold versus contrast curves by Legge and Foley (1980). The model predicts several prominent features of these curves, such as the "dipper" portion of the curve at low test contrasts and the linear rise (on a double logarithmic plot) of threshold at high test contrasts. The assumption that threshold corresponds to a fixed criterion increase in response, $\Delta R$, has also been used extensively to model contrast discrimination by Foley and his colleagues (Boynton \& Foley, 1999; Foley, 1994; Foley \& Chen, 1997; Foley \& Yang, 1991).

We modeled the data by making the assumption that $\sigma$ in the equations above was set by the arithmetic mean of the test and masking contrasts plus a resting half-saturation contrast, $c_{0}$. In other words, the half-saturation contrast was set by the timeaveraged contrast across at least one period of the $8-\mathrm{Hz}$ contrast alternation plus a small intrinsic noise contrast, $c_{0} \cdot{ }^{\prime}$ This can be modeled as shown in Equation 5.

$$
\sigma=c_{0}+0.5\left(c+c_{\text {mask }}\right) .
$$




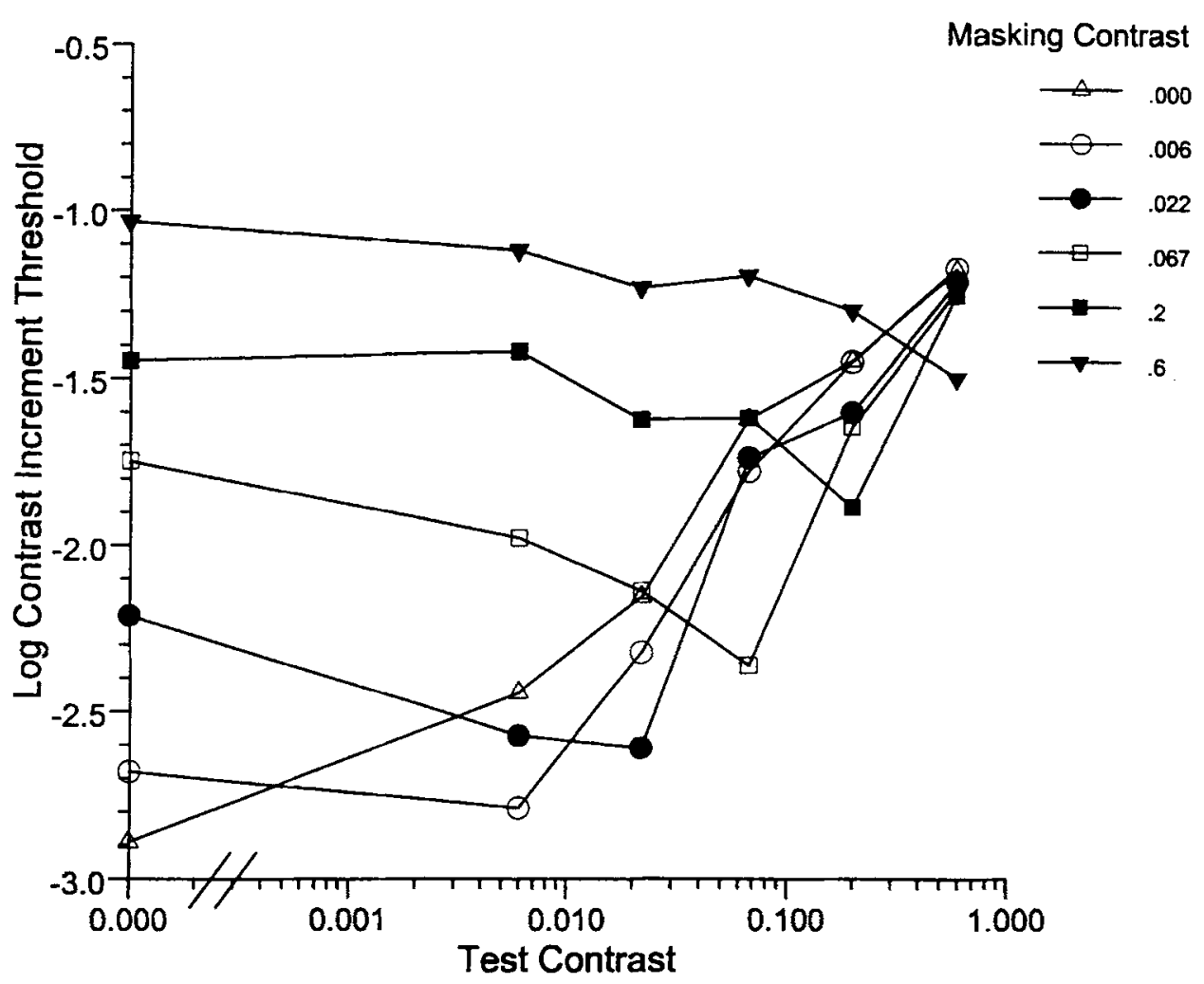

Figure 4. Contrast increment threshold versus contrast curves for Observer A.P. The curves have been extended for convenience over the $x$-axis break to a test contrast of zero. These data and the data shown in Figures 5, 6, and 7 for the other observers should be compared with the pattern of results predicted by the model and shown in Figure 3.

When Equation 5 is substituted into Equation 1, the following expression for the contrast versus response function is obtained:

$$
R(c)=R_{\max } \cdot\left[\frac{c^{n}}{c^{n}+\left[c_{0}+.5 \cdot\left(c+c_{\text {mask }}\right)\right]^{n}}\right] .
$$

To understand the behavior of the model as the masking contrast is changed, suppose that the masking contrast is increased by a factor of $k$ and that the resting noise contrast is negligible relative to the time-averaged contrast [i.e., $c_{0}<<.5\left(c+c_{\text {mask }}\right)$ ]. This latter assumption puts us in a stimulus regime in which the intrinsic noise is swamped by the time-averaged contrast, allowing us to set $c_{0}$ effectively to zero. Substitution of $k c_{\text {mask }}$ for $c_{\text {mask }}$ in Equation 6 and subsequent algebraic manipulation leads to Equation 7:

$$
R(c)=R_{\max } \cdot\left[\frac{\left(\frac{c}{k}\right)^{n}}{\left(\frac{c}{k}\right)^{n}+.5^{n}\left[\left(\frac{c}{k}\right)+c_{\text {mask }}\right]}\right] .
$$

Equation 7 shows that increasing the mask contrast by a factor of $k$ is equivalent to leaving the mask contrast fixed and scaling the test contrast by a factor of $1 / k$-that is, changing the gain with which contrast is processed. Contrasts that previously evoked a sizable response now produce much smaller responses in the presence of the higher contrast mask. This change in the mask contrast shifts the contrast versus response function along the contrast axis. Because both the test and increment contrasts are scaled by the same gain factor, the net effect of increasing the mask contrast and averaging it linearly with the test contrast to set the half-saturation contrast is a shift of the tvc function along a diagonal line on a double logarithmic plot. This can be seen in Figure 3 , which plots model tve curves for various test contrasts on the $x$-axis with each of the curves corresponding to a different masking contrast. These curves as defined in Equation 4 are simply diagonally shifted versions of each other, assuming that the resting noise contrast is negligible relative to the time-averaged contrast.

Thus, there is a particularly simple test of the hypothesis that contrast gain is set in this situation by averaging the test and masking contrasts. One of the tvc curves can be left plotted as usual. The other tvc curves can be shifted diagonally by the ratio of their masking contrasts to the masking contrast of the curve that was left unshifted. All of the data should then be fit by a single template tve curve if the hypothesis is correct. This should hold only over stimulus conditions in which the resting noise contrast can be effectively ignored. Wilson and Kim (1998) also used this strategy of diagonally shifting probe-on-flash contrast thresholds as a way of testing for the presence of a divisive contrast gain control mechanism.

Finally, we note that Equation 5 can be written more generally with different weights assigned to the test and mask contrasts in the averaging process. There is no a priori reason to think that the weights should be unequal given that the test and mask contrasts were displayed for equal durations $(62.5 \mathrm{msec})$ during each cycle. A conservative assumption is that the weights were equal. Changing the test:mask duty cycle of the $8-\mathrm{Hz}$ stimulation from $1: 1$ would allow an empirical test of this simple averaging assumption. 


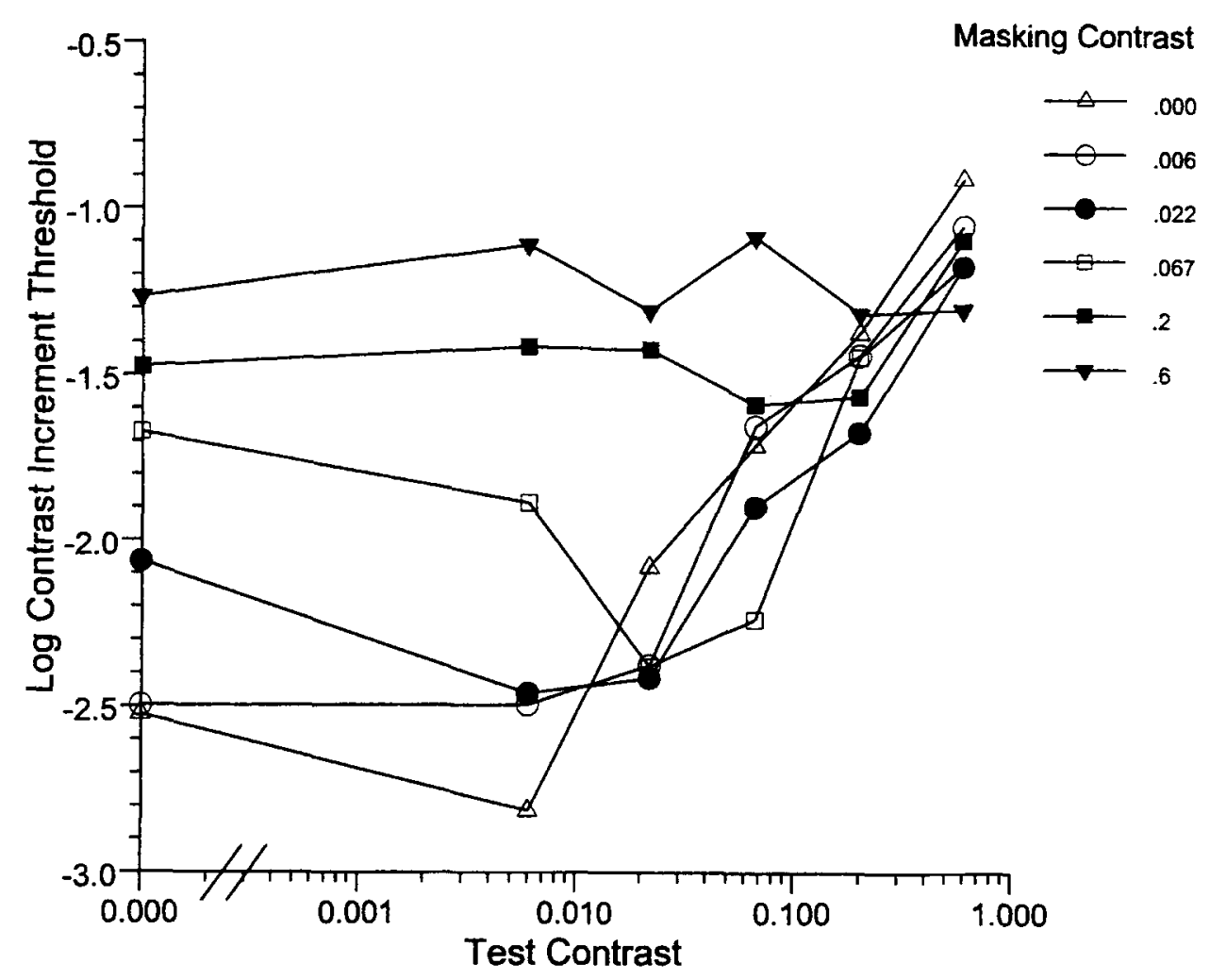

Figure 5. Same conventions as Figure 4 for Observer J.D.

\section{RESULTS}

Figures 4-7 show the data from the 4 observers. The test contrast is plotted on the $x$-axis and the contrast increment threshold is plotted on the $y$-axis on a double logarithmic plot. Each curve represents threshold versus test contrast with a different masking contrast. The pattern of results was similar across all of the observers. The threshold versus contrast curves shift up and to the right as the masking contrast is increased. Most of the curves, especially with the higher masking contrasts, show minima when the masking contrast is near the test contrast.

Table 1 shows the fits of the full model using Equations 4 and 5 and including a resting noise term for the 4 observers. There are several things to note regarding these parameter estimates. First, the fits of the model are very good, with just three parameters accounting for more than $85 \%$ of the variance among the 36 observed thresholds for each subject. Second, the resting noise contrasts, $c_{0}$, range from . $7 \%$ to $1.4 \%$-values that agree reasonably well with the value of $1.5 \%$ used by Ross and Speed (1991) in their model of contrast masking. Third, the $\Delta R$ estimates are similar across observers, indicating threshold with response increases ranging from $6 \%$ to $10 \%$. Finally, all of these observers show similar nonlinear exponents centering near 2.0. We note here that this value is theoretically consistent with Heeger's model of cortical contrast processing, which assumes a squaring nonlinearity. ${ }^{2}$ They are slightly lower than the values reported for cortical neurons by Albrecht and Hamilton $(1982$; mean exponent $=2.9)$ and by Sclar et al. $(1990$; mean exponent $=2.4$ ) for cortical neurons in macaque area V1. Legge and Foley (1980) used a threshold model with different exponents in the numerator and denominator of Equation 1. The denominator exponent was 2, and the numerator exponent that Legge and Foley used on their nonlinear transducer function was 2.4 . Our parameter estimates thus are similar to those used by Legge and Foley.

Recall that when the resting noise contrast can be assumed to be negligible relative to the time-averaged contrast, the model above, in which the gain is set by the time-averaged contrast, predicts that all of the threshold curves should be diagonally shifted versions of each other. To test the hypothesis, each observer's data have been replotted in Figures $8-11$ by diagonally shifting the tvc curves by the ratio of the masking contrast to .006 . The data with a masking contrast of .006 are left unshifted in each plot. We excluded the series of six thresholds collected for each observer with a zero contrast mask because of the assumption that the effect of the resting noise contrast should be small relative to the physical, timeaveraged contrast. The solid curve in each plot is the best fit of Equation 4, with the resting noise contrast, $c_{0}$, con- 


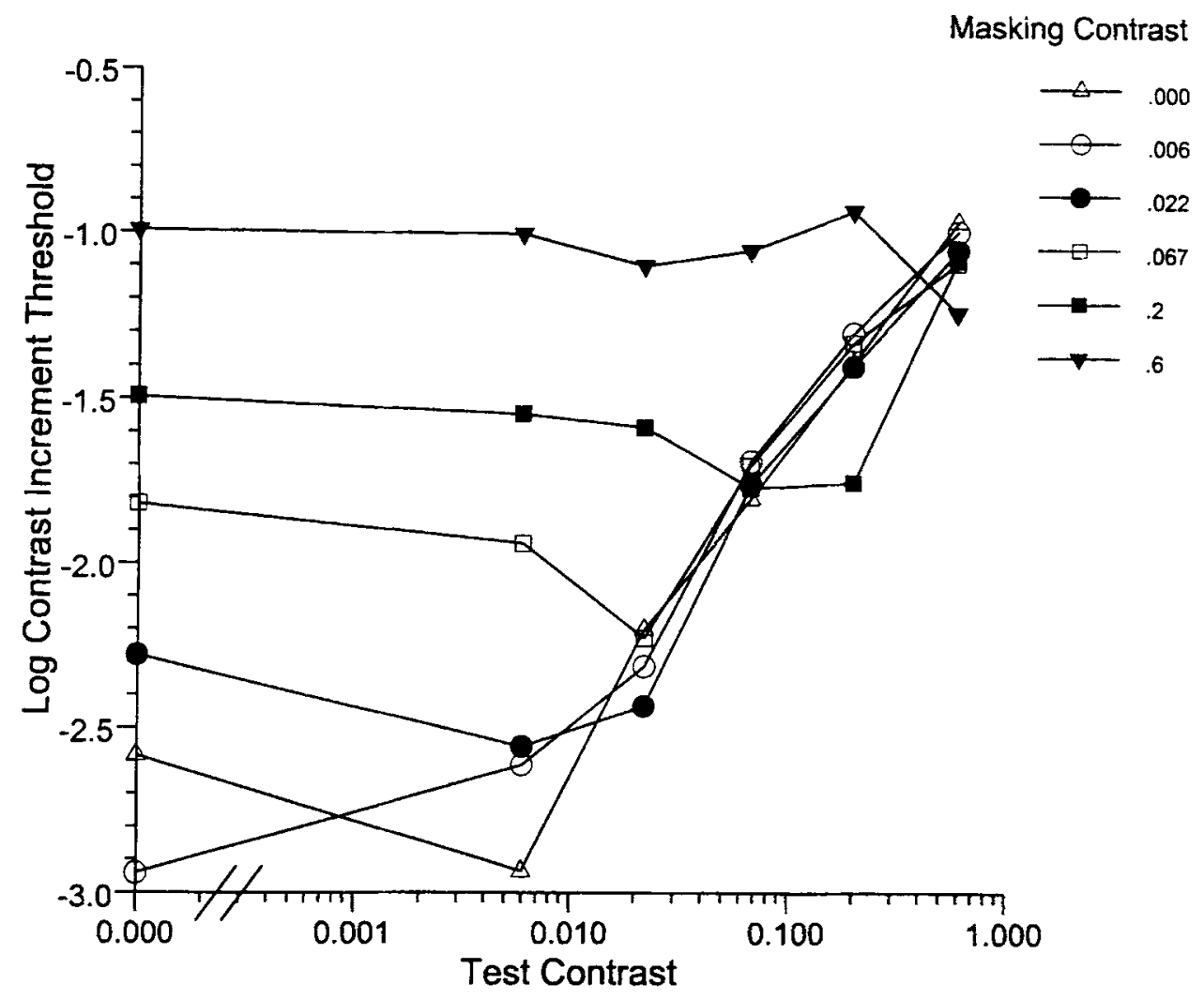

Figure 6. Same conventions as Figure 4 for Observer J.S.

strained to be zero. All of the fits were done, therefore, using the other 30 data points with nonzero masking contrasts.

Figures 8-11 show that when the data are diagonally shifted on a log-log plot by the ratios of their masking contrasts to .006 (the masking contrast of the unshifted curve), a single template tvc curve does a good job of capturing the variation in thresholds across the masking and test conditions. The only systematic deviation appears to be at high relative test contrasts, where observed thresholds are slightly lower than thresholds predicted from the best fitting tvc curve. To a first approximation, then, these data are consistent with the hypothesis that the threshold contrast increment depends on the linear average of the test and masking contrasts and with a model in which the half-saturation contrast shifts to match this time-averaged contrast. This approximation holds best when the resting or intrinsic noise contrast can be assumed to be small relative to the time-averaged contrast.

We also considered an alternative model that was based on inspection of the stimulus conditions shown in Figure 1 . Notice that a cue to the interval with the increment in contrast would be the depth of modulation when the test and masking contrasts are not equal. One alternative model of threshold would be that the threshold increment in contrast, $\Delta c$, is proportional to the depth of the contrast modulation plus some constant value required by noise in the discrimination. This model at first seems intuitively plausible because it predicts that the threshold curves all should show minima when the test and masking contrasts are equal because the depth of modulation is zero at that point. The depth of the contrast modulation in Figure 1 is equal to the difference between the test and masking contrasts. The data in Figures 4-7 can be used to rule out this alternative model of threshold. If this model were true, all of the tvc curves should dip to the same threshold $\Delta c$ when the test and masking contrasts are equal because at this point the depth of modulation is zero, and threshold would be determined solely by the noise in the system. It is obvious that this model cannot account for the data because the curves shift systematically to higher levels on the $y$-axis as the masking contrast is increased. It would be possible, perhaps, to rescue this alternative model by including additional ad hoc assumptions, but we did not pursue this endeavor because such a model is not theoretically motivated.

\section{DISCUSSION}

This experiment shows that contrast discrimination under temporally varying contrast conditions depends on the time-averaged contrast in the visual field. When a test contrast is alternated at $8 \mathrm{~Hz}$ with a masking contrast, the threshold contrast increment depends on the average of 


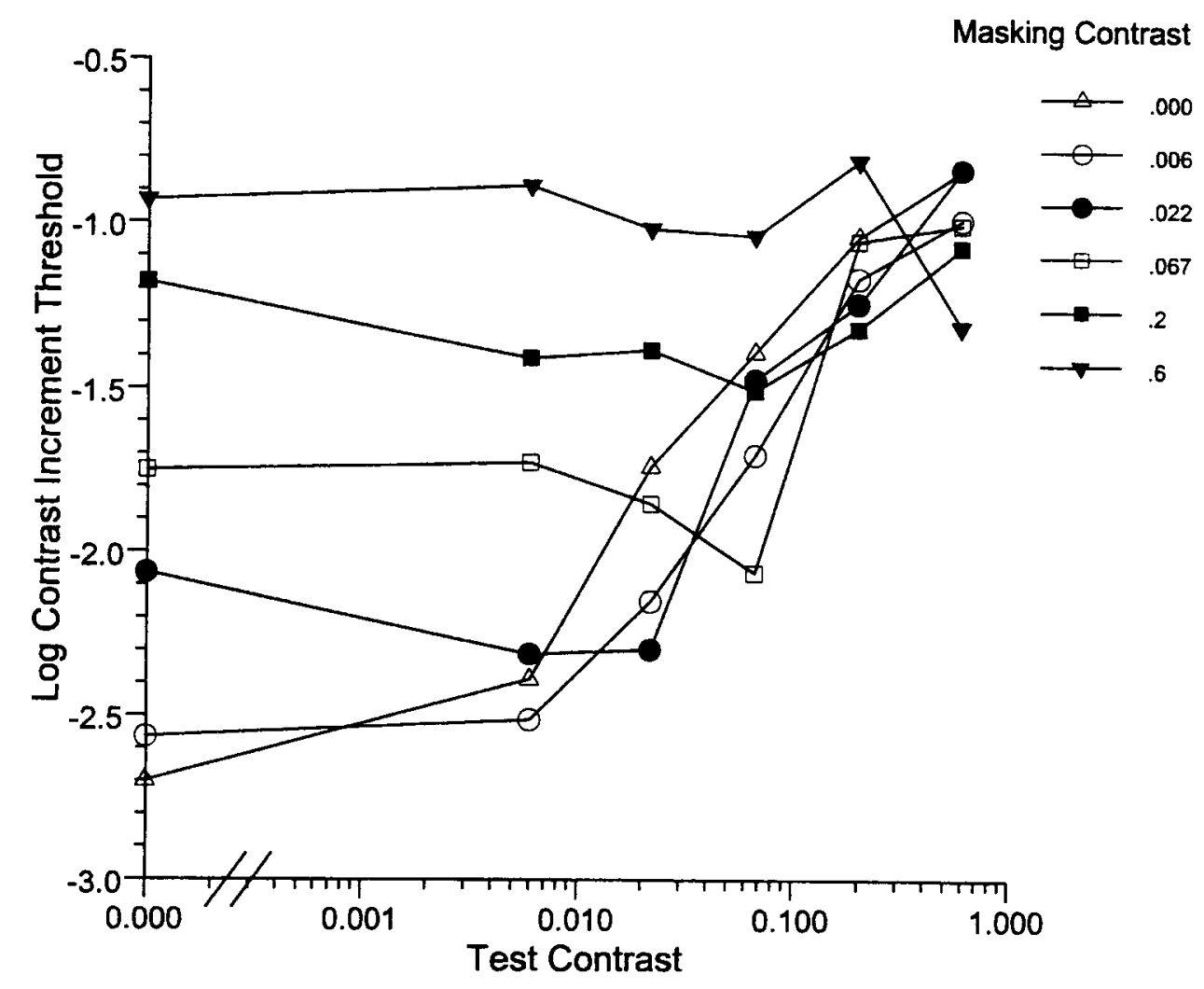

Figure 7. Same conventions as Figure 4 for Observer S.M.

these two contrasts. For a given masking contrast, threshold is generally lowest near the point at which the test contrast equals this masking contrast. As the difference between these two contrasts increases, the contrast increment threshold rises. The rise is steeper when the test contrast is greater than the masking contrast than it is when the difference is in the other direction.

We also ruled out another explanation for the data on the basis of discrimination of contrast modulation amplitude. Inspection of Figure 1 shows that as the contrast increment is changed, the depth of contrast modulation changes. Observers might have used such a cue to judge the interval with the greater contrast in the test grating. The data are not compatible, however, with such an explanation. On the assumption that threshold should be proportional simply to the difference between the test and mask contrasts (contrast modulation depth), one would have expected to see all thresholds dip to the same level when the test and mask contrasts were equal (zero contrast modulation). This was not the case. It is true that thresholds dipped in the region where the test and masking contrasts were equal, but the absolute levels of contrast had a large effect (as would be expected from previous contrast discrimination studies). Thresholds were not simply determined by the amplitude of the contrast modulation.
The hypothesis that we set out to test with these data was confirmed. The threshold versus contrast curves could be aligned by sliding them diagonally on doublelogarithmic plots by an amount that depended simply on the ratio of the masking contrasts, assuming that the effect of the resting noise contrast was negligible. This behavior was predicted by a simple model of contrast discrimination based on ideal, nonlinear contrast versus response functions (Albrecht \& Hamilton, 1982; Heeger, 1992a, 1992b). By adding the assumption to this model that the half-saturation contrasts of these ideal crf's were determined by the linear average of the test and masking contrasts, it was possible to predict this behavior for the tvc curves. As shown in Heeger (1992b) and above, changes in the half-saturation contrast of a cortical neuron are equivalent to sliding the crf along a logarithmic contrast axis. In other words, such changes effectively alter the gain with which contrast is processed. Doubling the half-saturation contrast is equivalent to halving all of the input contrasts. Our results show that this form of contrast gain control averages contrast over at least $125 \mathrm{msec}$ in adjusting sensitivity to differential contrast.

The model curves generated from Equations 4 and 5 provided good fits to the data from the 4 observers. The one possible exception to this statement is at high relative contrast levels between the mask and the test contrasts. 


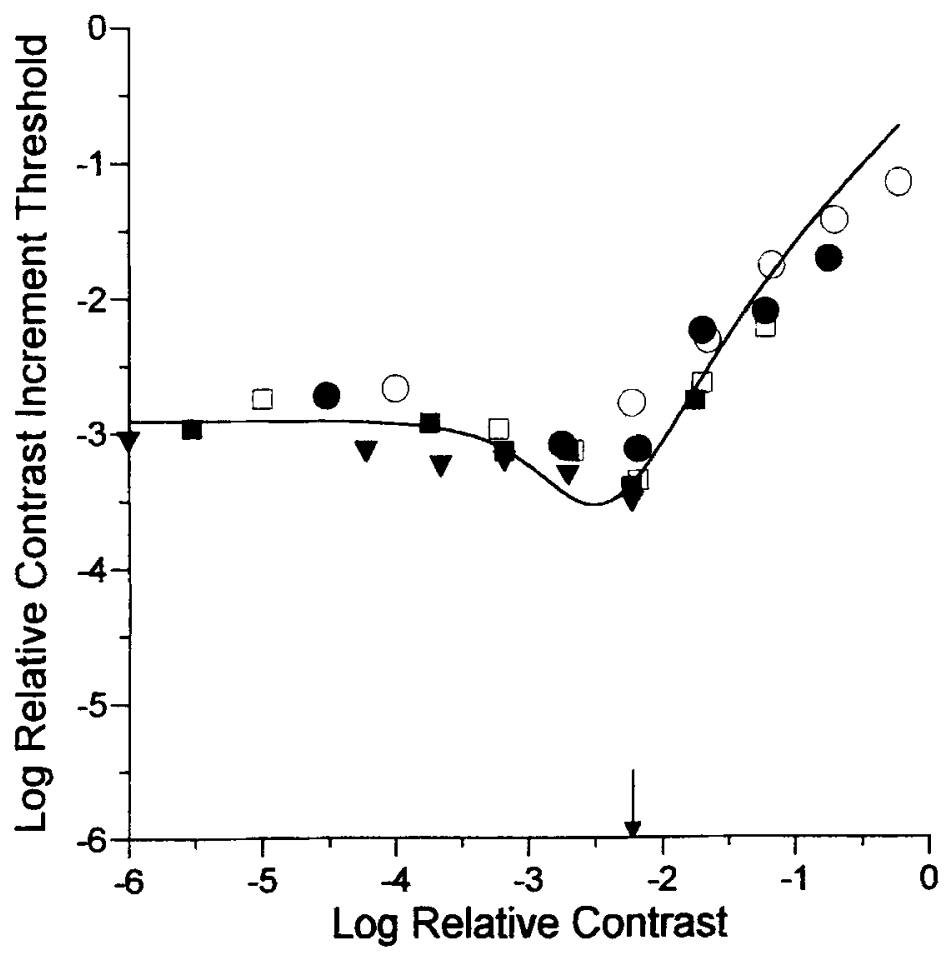

Figure 8. Replot of data in Figure 4 for Observer A.P. The symbols correspond to the masking contrast series shown in Figure 4. The data for a masking contrast of .006 have been left unshifted (arbitrary decision). All of the other data with nonzero masking contrasts have been shifted diagonally by the ratio of the masking contrast to $\mathbf{. 0 0 6}$. The solid curve is the best fit of Equations 4 and 5 (assuming negligible resting noise contrast) to the data from all conditions with nonzero masking contrasts $(n=30$ data points). The arrow on the $\boldsymbol{x}$-axis shows the point at which the test and masking contrasts were equal. See text for details of curve-fitting procedures.

Examination of the best fitting curves in Figures 8-11 shows that incremental sensitivity was slightly better than was predicted by the model. The effect is slight, although it appears systematic. One way to accommodate this discrepancy would be to allow the nonlinear exponents in Equation 1 to differ in the numerator and the denominator, as Legge and Foley (1980) and Boynton and Foley (1999) did. Given that the data conform reasonably well to the simple model that we used with the nonlinear exponents in the numerator and denominator constrained to be equal, we did not pursue this additional step in fitting the data.

Boynton, Demb, Glover, and Heeger (1999) also recently modeled human contrast discrimination using a similar model with additive internal noise. Boynton et al. found that psychophysical contrast increment thresholds were predicted well by assuming that these observers detected the increment in the presence of this noise whenever the pooled activity of a population of neurons increased by a criterion amount (similar to our $\Delta R$ parameter). As noted, this assumption about threshold corresponding to a fixed criterion increase in response has been used many times previously to model contrast discrimination (Boynton \& Foley, 1999; Foley, 1994; Foley \& Chen, 1997; Foley \& Yang, 1991; Legge \& Foley, 1980). The activity of neurons in visual cortical areas VI, V2d, V3d, and V3A as measured by functional magnetic resonance imaging (fMRI) was also consistent with this simple contrast discrimination model.

It is worth noting that the effect of the intervening masker being averaged with the test contrast to determine threshold is probably highly dependent on the temporal frequency of the alternation of the test and masker. At very high temporal frequencies, above the CFF, for example, the test and mask contrasts would be effectively averaged at the level of the photoreceptors, so results similar to those that we observed would not be at all unexpected. At very low temporal frequencies, there would probably be no effect of the masker because sufficient time would elapse before the onset of the mask to allow gain to be set optimally for the test contrast. We tested with an $8-\mathrm{Hz}$ alternation rate and found that the test and masker contrast 


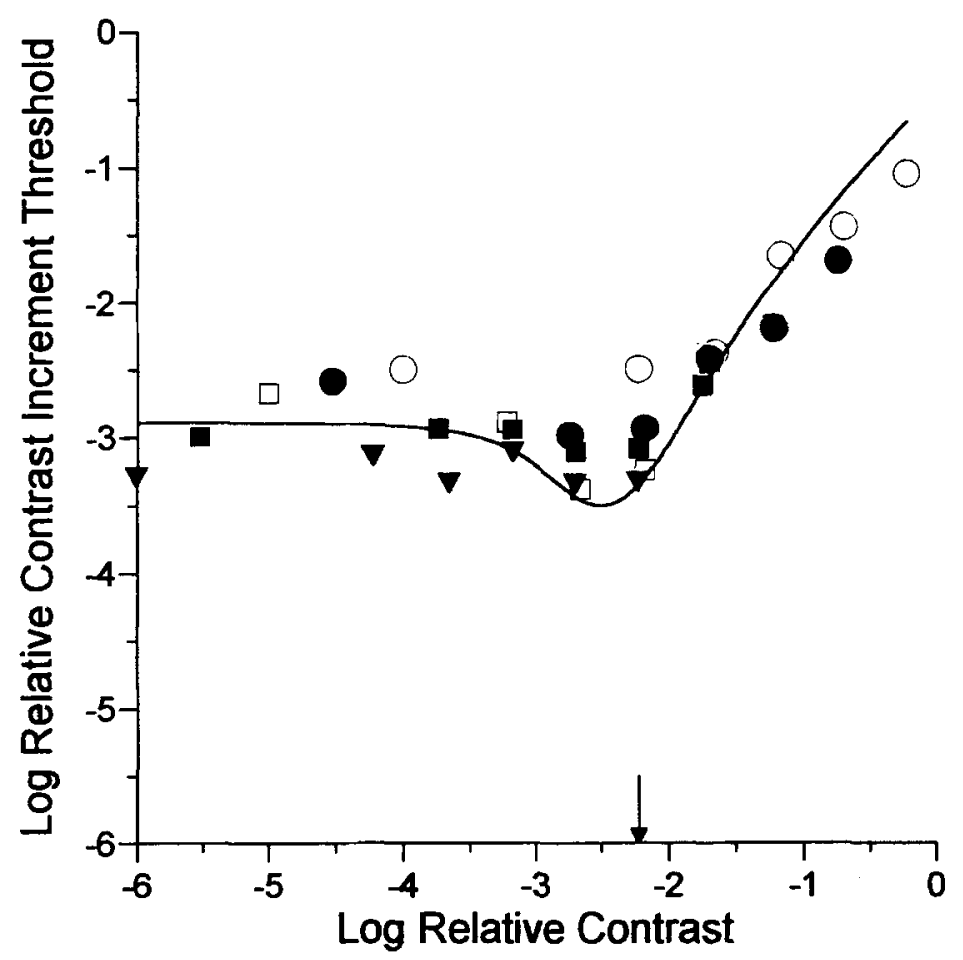

Figure 9. Same conventions as Figure 8 for Observer J.D.

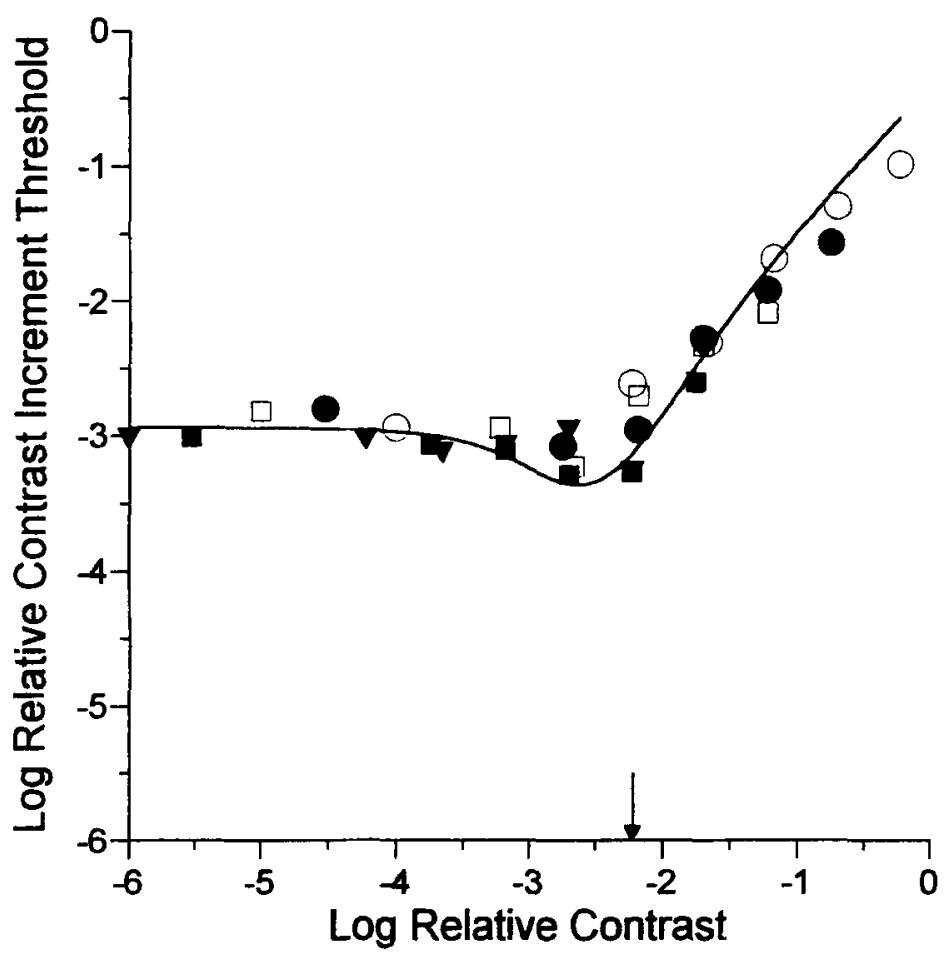

Figure 10. Same conventions as Figure 8 for Observer J.S. 


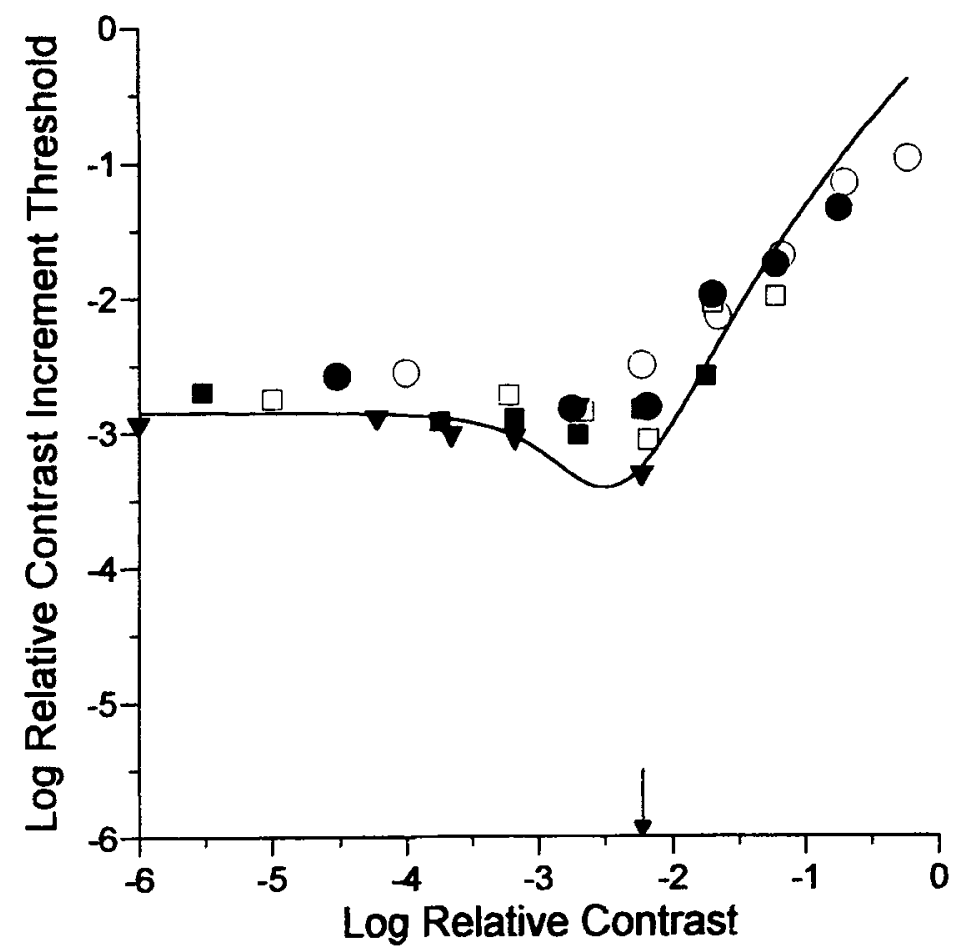

Figure 11. Same conventions as Figure 8 for Observer S.M.

were effectively averaged. Such a result implies that the mechanism that determines contrast gain averages contrast over at least a period of $125 \mathrm{msec}$.

Heeger (1992b) noted that such a gain control mechanism would need to average contrast over some finite period to avoid temporal instabilities in contrast processing, although this time constant was left unspecified in Heeger's (1992b) model. Geisler and Albrecht (1992) showed data from cortical neurons compatible with a fast-acting gain control process that scales input contrast by the average local contrast. Bonds $(1989,1993)$ has also shown that responses from visual cortical neurons in cats adjust to the recent history of contrast within their receptive fields. The time constants for this gain control in Bonds's data range over periods from tens to hundreds of milliseconds. The neurophysiological data, therefore, argue for some form of contrast gain control, although they do not allow the time constant to be specified precisely.

Psychophysical evidence compatible with this type of contrast gain control and estimates of its time constant come from several sources. The time constant was approximately $60 \mathrm{msec}$ in Wilson and Humanski's model (1993), with the integration process taking approximately $200 \mathrm{msec}$ to reach $96 \%$ completion. Masks required durations of approximately $150 \mathrm{msec}$ in masking data to be fully effective in Wilson (1993) and in Wilson and Kim (1998). As noted, Bowen and Wilson (1994) showed that threshold elevation depended strongly on the SOA between a preceding contrast mask and a target. Threshold was elevated substantially at short SOAs, presumably before contrast gain control could adjust sensitivity. Threshold elevation dropped rapidly over the first $60-200 \mathrm{msec}$.

Table 1

Parameter Estimates from

Equations 4 and 5 for Each Observer

\begin{tabular}{lcccc}
\hline Observer & $n$ & $\left(\Delta R / R_{\max }\right) \times 100 \%$ & $\begin{array}{c}\text { Percent } \\
\text { Variance Explained }\end{array}$ & $\begin{array}{c}\text { Resting } \\
\text { Noise Contrast, } c_{0}\end{array}$ \\
\hline A.P. & 2.15 & 5.85 & 86.0 & .010 \\
J.D. & 2.06 & 6.52 & 89.5 & .014 \\
J.S. & 2.05 & 7.30 & 92.4 & .007 \\
S.M. & 1.99 & 9.96 & 85.4 & .011 \\
\hline
\end{tabular}

*Model fits based on all 36 data points with all values represented logarithmically using the Minerr function in Mathcad. Zero contrasts were represented as -6.0 for purposes of model fitting. 
All of these psychophysical studies support the conclusion that contrast may be averaged over approximately 100-200 msec to set contrast gain. We note here, as did Wilson and Kim (1998), that natural saccadic frequencies are in the range of three to four per second, thus allowing such a mechanism to adjust sensitivity to each new fixation depending on the local average contrast.

\section{REFERENCES}

Albrecht, D. G., \& GeisLer, W. S. (1991). Motion selectivity and the contrast-response function of simple cells in the visual cortex. Visual Neuroscience, 7, 531-546.

Albrecht, D. G., \& Hamilton, D. B. (1982). Striate cortex of monkey and cat: Contrast response function. Journal of Neurophysiology, 48, 217-237.

Bonds, A. B. (1989). Role of inhibition in the specification of orientation selectivity of cells in the cat striate cortex. Visual Neuroscience, 2, 41-55.

BonDS, A. B. (1993). The encoding of cortical contrast gain control. In R. Shapley \& D. M.-K. Lam (Eds.), Contrast sensitivity (pp. 215230). Cambridge: MIT Press.

BOWEN, R. W., \& WILSON, H. R. (1994). A two-process analysis of pattern masking. Vision Research, 34, 645-657.

Boynton, G. M., Demb, J. B., Glover, G. H., \& Heeger, D. J. (1999). Neuronal basis of contrast discrimination. Vision Research, 39, 257 . 269.

BOYNTON, G. M., \& FolEy, J. M. (1999). Temporal sensitivity of human luminance pattern mechanisms determined by masking with temporally modulated stimuli. Vision Research, 39, 1641-1656.

DANNEmiller, J. L., \& STEPhens, B. R. (1998). Contrast gain control in psychophysical contrast discrimination. Perception \& Psychophysics, 60, 1153-1163.

FOLEY, J. M. (1994). Human luminance pattern-vision mechanisms: Masking experiments require a new model. Journal of the Optical Society of America A, 11, 1710-1719.

Foley, J. M., \& CHEN, C. C. (1997). Analysis of the effect of pattern adaptation on pattern pedestal effects: A two-process model. Vision Research, 37, 2779-2788.

FOLEY, J. M., \& YANG, Y. D. (1991). Forward pattern masking: Effects of spatial frequency and contrast. Journal of the Optical Society of America A, 8, 2026-2037.

GEISLER, W. S., \& ALBRECHT, D. G. (1992). Cortical neurons: Isolation of contrast gain control. Vision Research, 32, 1409-1410.

Geisler, W. S., \& AlbRecht, D. G. (1997). Visual cortex neurons in monkeys and cats-Detection, discrimination and identification. $V i$ sual Neuroscience, 14, 897-919.
GeORGESON, M. A., \& GEORGESON, J. M. (1987). Facilitation and masking of briefly presented gratings: Time-course and contrast dependence. Vision Research, 27, 369-379.

HeEger, D. J. (1992a). Half-squaring in responses of cat striate cells. Visual Neuroscience, 9, 427-443.

HeEger, D. J. (1992b). Normalization of cell responses in cat striate cortex. Visual Neuroscience, 9, 181-197.

HeEger, D. J. (1994). The representation of visual stimuli in primary visual cortex. Current Directions in Psychological Science, 3, 159-163.

Hubel, D. H., \& WIESEL, T. N. (1968). Receptive fields and functional architecture of monkey striate cortex. Journal of Physiology, 195, 215-243.

LegGe, G. E., \& Foley, J. M. (1980). Contrast masking in human vision. Journal of the Optical Society of America, 70, 1458-1471.

NAKA, K. I., \& RuShTON, W. A. H. (1966). S-potentials from luminosity units in the retina of fish (Cyprinidae). Journal of Physiology, 185, 587-599.

Ross, J., \& SPEED, H. D. (1991). Contrast adaptation and contrast masking in human vision. Proceedings of the Royal Society of London: Series $B, 246,61-69$.

SClar, G., Maunsell, J. H. R., \& Lennie, P. (1990). Coding of image contrast in central visual pathways of the macaque monkey. Vision Research, 30, 1-10.

WILson, H. R. (1993). Nonlinear processes in visual pattern discrimination. Proceedings of the National Academy of Sciences, 90, 9785 9790.

Wilson, H. R., \& Humanski, R. (1993). Spatial frequency adaptation and contrast gain control. Vision Research, 33, 1133-1149.

WILSON, H. R., \& KIM, J. (1998). Dynamics of a divisive gain control in human vision. Vision Research, 38, 2735-2741.

\section{NOTES}

1. We thank an anonymous reviewer for pointing out the necessity of including this intrinsic noise term in the model. When mask and test contrasts are set to zero, the model described by Equation 4 predicts a threshold contrast of zero. Including a resting noise contrast as shown in Equation 5 allows a nonzero gain to be determined. As explained in the text, this intrinsic noise parameter becomes less influential as the time-averaged contrast increases.

2. We note here that when the model fitting was done using only the 30 data points from nonzero mask contrast conditions diagonally translated as described in the text and with the noise parameter, $c_{0}$, constrained to be zero, the estimated exponents were more variable and somewhat higher, ranging from 2.6 to 3.4 .

(Manuscript received August 11, 1999; revision accepted for publication August 3, 1999.) 